\title{
L-2-Oxothiazolidine-4-Carboxylic Acid Reverses Endothelial Dysfunction in Patients with Coronary Artery Disease
}

\author{
Joseph A. Vita, ${ }^{\star}$ Balz Frei, ${ }^{*}$ Monika Holbrook, ${ }^{*}$ Noyan Gokce, ${ }^{\star}$ Cynthia Leaf, ${ }^{\ddagger}$ and John F. Keaney, Jr. ${ }^{\star}$ \\ *Evans Memorial Department of Medicine, Cardiology Section and Whitaker Cardiovascular Institute, Boston University School of \\ Medicine, Boston, Massachusetts 02118; and ${ }^{\ddagger}$ Transcend Therapeutics, Inc., Cambridge, Massachussetts 02139
}

\begin{abstract}
The effective action of endothelium-derived nitric oxide (EDNO) is impaired in patients with atherosclerosis. This impairment has been attributed in part to increased vascular oxidative stress. EDNO action is improved by administration of ascorbic acid, a water-soluble antioxidant. Ascorbic acid is a potent free-radical scavenger in plasma, and also regulates intracellular redox state in part by sparing cellular glutathione. We specifically investigated the role of intracellular redox state in EDNO action by examining the effect of L-2-oxo-4-thiazolidine carboxylate (OTC) on EDNO-dependent, flow-mediated dilation in a randomized double-blind placebo-controlled study of patients with angiographically proven coronary artery disease. OTC augments intracellular glutathione by providing substrate cysteine for glutathione synthesis. Brachial artery flow-mediated dilation was examined with high-resolution ultrasound before and after oral administration of $4.5 \mathrm{~g}$ of OTC or placebo in 48 subjects with angiographically documented coronary artery disease. Placebo treatment produced no change in flowmediated dilation $(7.0 \pm 3.9 \%$ vs. $7.2 \pm 3.7 \%)$, whereas OTC treatment was associated with a significant improvement in flow-mediated dilation ( $6.6 \pm 4.4 \%$ vs. $11.0 \pm 6.3 \% ; P=0.005)$. OTC had no effect on arterial dilation to nitroglycerin, systemic blood pressure, heart rate, or reactive hyperemia. These data suggest that augmenting cellular glutathione levels improves EDNO action in human atherosclerosis. Cellular redox state may be an important regulator of EDNO action, and is a potential target for therapy in patients with coronary artery disease. (J. Clin. Invest. 1998. 101:1408-1414.) Key words: antioxidants • nitric oxide • blood vessels • endothelium • glutathione
\end{abstract}

\section{Introduction}

Normal vascular homeostasis is dependent upon endothelial elaboration of paracrine factors that prevent both platelet adhesion to the endothelial surface and inappropriate vasospasm. One important factor responsible for these functions is

Address correspondence to J.A. Vita, Section of Cardiology, Boston Medical Center, 88 E. Newton St., Boston, MA 02118. Phone: 617638-8701; FAX: 617-638-8712; E-mail: jvita@acs.bu.edu B. Frei’s present address is Linus Pauling Institute at Oregon State University, Weniger Hall, Corvallis, OR 97331.

Received for publication 10 July 1997 and accepted in revised form 30 December 1997.

J. Clin. Invest.

(C) The American Society for Clinical Investigation, Inc. 0021-9738/98/03/1408/07 \$2.00

Volume 101, Number 6, March 1998, 1408-1414

http://www.jci.org endothelium-derived nitric oxide (EDNO). ${ }^{1}$ Abnormalities in the action and metabolism of EDNO have been described in association with established coronary artery disease $(1,2)$ or risk factors for coronary artery disease (3). This impairment in EDNO action likely contributes to the development of clinically significant vascular events (4).

The precise mechanism of impaired EDNO action in atherosclerosis is not clear, although emerging evidence suggests that increased vascular oxidative stress is an important component of this phenomenon (5). Important sources of oxidative stress in atherosclerosis and hypercholesterolemia include superoxide anion and lipid peroxidation. For example, animal models of atherosclerosis and hypercholesterolemia are characterized by excess vascular production of superoxide $(6,7)$, and superoxide is known to inactivate $\operatorname{EDNO}(8,9)$. Lipid peroxidation in the vascular wall results in local accumulation of oxidized low-density lipoprotein (ox-LDL; 10) that may inhibit the production of EDNO (11) and accelerate EDNO degradation (12).

This link between excess vascular oxidative stress and impaired EDNO action is supported by a number of studies examining the effect of improved antioxidant defenses on EDNO-mediated control of arterial relaxation. Treating cholesterol-fed rabbits with polyethylene glycol-conjugated superoxide dismutase (SOD; 13), $\alpha$-tocopherol (14), or probucol $(7,15)$ improves EDNO-mediated arterial relaxation. In patients with coronary artery disease, antioxidant treatment with probucol combined with cholesterol reduction improves endothelial function more effectively than does cholesterol reduction alone (16).

It is important to realize that enzymatic antioxidants such as SOD and lipid-soluble agents like $\alpha$-tocopherol represent only part of the vascular wall antioxidant defenses. A significant amount of cellular antioxidant protection is afforded by water-soluble antioxidants such as ascorbic acid and glutathione (17). In this regard, acute infusion of supraphysiologic concentrations $(\sim 10 \mathrm{mM}$ ) of ascorbic acid improve EDNOmediated arterial relaxation in patients with diabetes (18), a history of cigarette smoking (19), or hypercholesterolemia (20). In patients with established coronary artery disease (CAD), we observed an acute (2-h) improvement in EDNOmediated brachial artery dilation after a single 2-g oral dose of ascorbic acid that raises plasma ascorbate concentration 2.5fold within the physiologic range $(50-150 \mu \mathrm{M} ; 21)$.

Although ascorbic acid is the main water-soluble antioxidant in human plasma, it also plays a central role in regulation of intracellular redox state (17). Both ascorbic acid and GSH are present in millimolar concentrations within the cell cytosol,

1. Abbreviations used in this paper: CAD, coronary artery disease; EDNO, endothelium-derived nitric oxide; GSNO, S-nitroso-glutathione; DTDP, 2,2'-dithiodipyridine; NO, nitric oxide; NOS, nitric oxide synthase; OTC, L-2-oxo-4-thiazolidine carboxylate. 
and serve both to scavenge free radical species and to detoxify reactive oxygen species that are produced by normal oxidative metabolism (17). A recent study demonstrated reduced arterial tissue GSH in experimental atherosclerosis (22). Despite the importance of these species for cellular function in normal and pathological states, the extent to which intracellular redox state contributes to EDNO-mediated control of vascular tone is not clear at present. The purpose of this study was to examine the effect of increasing intracellular glutathione on abnormal EDNO-mediated arterial relaxation in patients with established coronary artery disease.

\section{Methods}

Pharmacokinetics. In this study, we used L-2-oxo-4-thiazolidine carboxylate (OTC; Procysteine ${ }^{\mathrm{TM}}$; Transcend Therapeutics, Inc., Cambridge, MA) to increase intracellular GSH levels. This compound is readily transported into cells and converted into cysteine by the intracellular enzyme 5-oxoprolinase $(23,24)$. Increased intracellular cysteine availability leads to increased intracellular GSH levels in a variety of tissues, including arteries and veins $(25,26)$. In a human study, Porta and colleagues reported a $167 \pm 60 \%$ increase in lymphocyte cysteine concentration and a $79 \pm 9 \%$ increase in lymphocyte glutathione concentration after an oral dose of OTC $(0.15 \mathrm{mmol} / \mathrm{kg} ; 27)$. The peak intracellular cysteine and glutathione concentrations occurred $1.5-3$ and 2-4 h after the oral OTC dose, respectively (27). In pharmacokinetic studies with six healthy volunteers using $0.5-4.5 \mathrm{~g}$ of oral OTC, bioavailability was maximal with the $4.5 \mathrm{~g}$ oral dose with $92 \%$ of the dose available to the systemic circulation (data provided by Transcend Therapeutics, Inc.). After oral administration of $4.5 \mathrm{~g}$ of OTC, mean plasma OTC levels peaked $2.5 \mathrm{~h}$ after administration $(451 \pm 164 \mu \mathrm{M})$. Peak whole blood cyst(e)ine levels $(118 \mu \mathrm{M})$ were reached $4 \mathrm{~h}$ after dose administration, and were approximately double baseline levels $(66 \mu \mathrm{M}$; data provided by Transcend Therapeutics, Inc.). Based on this information, we chose to administer $4.5 \mathrm{~g}$ of oral OTC and determine vascular function $4 \mathrm{~h}$ after administration when the maximal drug effect was observed.

Patient population. Patients referred to the cardiology service at Boston Medical Center were screened for enrollment, and those with significant coronary artery disease were eligible for study. A detailed medical history was obtained. The presence of coronary artery disease was confirmed angiographically by the presence of at least one coronary stenosis $>50 \%$. The extent of coronary artery disease was graded by an established scoring system (28). All subjects gave informed consent, and the study was conducted in accordance with the policies and procedures of the Institutional Review Board of Boston Medical Center.

All vasoactive medications were withheld for at least $12 \mathrm{~h}$ before study, and all long-acting vasoactive compounds were withheld for at least $24 \mathrm{~h}$. Patients with uncontrolled hypertension, congestive heart failure, unstable angina, or any other conditions that would preclude safely withholding vasoactive medications were excluded from the study. Patients were also excluded if they had taken antioxidant vitamin supplements, estrogen replacement therapy, $N$-acetylcysteine, or cysteine supplements within $2 \mathrm{mo}$, or if they had a history of lactose intolerance or alcohol abuse. All patients were taking aspirin (325 $\mathrm{mg} / \mathrm{d}$ ) at the time of the study. Subjects were asked not to smoke for $24 \mathrm{~h}$ before the study. This study was randomized, double-blinded, and placebo-controlled. Subjects were consecutively assigned treatment using a randomization scheme provided by the manufacturer. The treatment assignments were unknown to all personnel involved in the performance of data collection and analysis until completion of the study.

Study protocol. EDNO action was assayed as endotheliumdependent, flow-mediated dilation of the brachial artery using highresolution vascular ultrasound according to previously established methodology $(21,29,30)$. Using an ultrasound system equipped with a $7.5-\mathrm{mHz}$ linear array transducer (140A; Toshiba America Medical Systems, Tustin, CA), two-dimensional and pulsed-Doppler blood flow images were obtained $2-20 \mathrm{~cm}$ cephalad to the antecubital crease with the patient resting supine in a quiet setting. For each patient, the transducer position was marked, and pre- and posttreatment images were obtained in the same location. Hyperemia was induced by inflating a narrow-width blood pressure cuff on the proximal portion of the arm to occlude arterial flow (at least $200 \mathrm{~mm} \mathrm{Hg}$ ) for $5 \mathrm{~min}$, and then rapidly deflating the cuff. Flow-velocity signal was recorded for $15 \mathrm{~s}$ after cuff release, and two-dimensional images were recorded starting $60 \mathrm{~s}$ after cuff release. Brachial artery dilation induced by this protocol was previously demonstrated to depend on nitric oxide (NO) synthesis (30).

Patients were then treated with either OTC ( $4.5 \mathrm{~g}$ orally) or identical placebo capsules. $4 \mathrm{~h}$ after treatment, the two-dimensional and pulsed Doppler measurements were repeated before and after reactive hyperemia. After an additional 10-min rest period, two-dimensional images were again obtained before and 3 min after administration of sublingual nitroglycerin $(0.4 \mathrm{mg})$. If the subject had a history of headache or other adverse reaction to nitroglycerin, this portion of the protocol was omitted. Before receiving study medication, vital signs were measured and a blood sample was obtained for analysis of serum lipids and glucose, plasma ascorbic acid, and whole blood cyst(e)ine. $4 \mathrm{~h}$ after treatment, vital signs were repeated and a second blood sample was obtained for cyst(e)ine and ascorbic acid determination.

Brachial arterial image and Doppler velocity profile analysis. Using ECG gating, ultrasound images were digitized on-line using a computer (Macintosh Quadra $840 \mathrm{AV}$ ) containing a digitizing board (LG-3; Scion Corp., Frederick, MD) and stored for subsequent analysis. Three images from three cardiac cycles were digitized for each of six conditions: before and during hyperemia at baseline, before and during hyperemia $4 \mathrm{~h}$ after study medication, and before and after nitroglycerin. In each patient, a $10-20 \mathrm{~mm}$ brachial artery segment was identified for analysis by use of anatomic landmarks. Average segment diameter for each image was determined in a blinded manner as previously described (21). Brachial artery flow-mediated dilation and extent of reactive hyperemia were determined as previously described $(21,31)$. Peak hyperemic blood flow was estimated as the product of vessel cross-sectional area, flow velocity, and heart rate using the cardiac cycle with the largest flow velocity integral after cuff deflation. The percent increase in blood flow during hyperemia was expressed as the percent increase in flow from baseline. The reproducibility of this method has been described in detail (31).

Biochemical analyses. Serum levels of total cholesterol, triglycerides, and glucose were determined by the Boston Medical Center clinical laboratory (model 717 automated analyzer; Hitachi Instruments, Indianapolis, IN). HDL cholesterol was similarly determined after phosphotungstic acid- $\mathrm{MgCl}_{2}$ precipitation of apo B-containing lipoproteins (32). LDL cholesterol was calculated using the Friedewald formula (33). Plasma ascorbic acid concentration was determined by paired-ion, reverse-phase HPLC with electrochemical detection as described (34). Whole blood cysteine was determined using monobromobimane derivitization and HPLC with fluorescence detection as described by Fiskerstrand and colleagues (35).

Statistical analysis. All data are presented as mean \pm SD unless otherwise indicated. Baseline clinical characteristics, vessel diameter, and blood flow were compared by the unpaired $t$ test or the Pearson Chi-square test as appropriate. The effect of treatment on baseline blood vessel diameter, brachial artery dilation, and hyperemic flow were compared using repeated-measures ANOVA with a posthoc Student Newman-Keuls comparison. Statistical analysis was performed with SPSS for Windows 6.1 software (SPSS Inc., Chicago, IL).

\section{Results}

Baseline characteristics. A total of 57 subjects were enrolled into the study. Nine subjects were excluded before the data 
Table I. Clinical Characteristics of Study Population

According to Treatment Assignment

\begin{tabular}{lcc}
\hline \multicolumn{1}{c}{ Parameter } & Placebo & OTC \\
\hline No. of subjects & 24 & 24 \\
Age (yr) & $55 \pm 10$ & $58 \pm 9$ \\
Female (\%) & 29 & 13 \\
Height (cm) & $175 \pm 8$ & $171 \pm 10$ \\
Weight (kg) & $90 \pm 16$ & $83 \pm 14$ \\
Total cholesterol (mg/dl) & $210 \pm 45$ & $206 \pm 42$ \\
HDL cholesterol (mg/dl) & $36 \pm 6$ & $37 \pm 8$ \\
LDL cholesterol (mg/dl) & $136 \pm 33$ & $134 \pm 31$ \\
Triglycerides (mg/dl) & $180 \pm 103$ & $177 \pm 85$ \\
Fasting glucose (mg/dl) & $116 \pm 35$ & $110 \pm 27$ \\
History of diabetes mellitus (\%) & 21 & 8 \\
History of smoking (\%) & 75 & 83 \\
Family history of premature CAD & 54 & 38 \\
History of hypertension & 58 & 54 \\
Current lipid-lowering therapy (\%) & 29 & 42 \\
Coronary atherosclerosis score (0-9) & $3.7 \pm 1.5$ & $4.2 \pm 1.7$ \\
& & \\
\hline
\end{tabular}

All data are mean \pm SD unless otherwise indicated. Clinical characteristics were derived from the patients' medical history. Lipid parameters were determined from a fasting blood sample. The coronary atherosclerosis score was determined as described by Hamsten and colleagues and expressed on a scale of 0 (no evidence of atherosclerosis) -9 (extensive disease in each of 15 coronary segments; 28 ).

were unblinded, eight were excluded because of poor image quality or missing brachial artery data, and one subject was excluded because of unrecognized systemic infection. The clinical characteristics of the 48 remaining subjects is contained in Table I. There were no significant differences in baseline characteristics based upon treatment assignment.

Brachial artery responses. The baseline brachial artery flow-mediated dilation was similar in the placebo and OTC groups (7.0 $\pm 3.8 \%$ vs. $6.5 \pm 4.3 \%$, respectively; $P=$ NS; Fig. 1$)$. Administration of the placebo was not associated with a significant change in flow-mediated brachial artery dilation over $4 \mathrm{~h}$ $(7.0 \pm 3.8 \%$ vs. $7.2 \pm 3.7 \%, P=\mathrm{NS})$. In contrast, treatment with OTC was associated with a significant improvement in brachial artery flow-mediated dilation from $6.5 \pm 4.3$ to $11.0 \pm$ $6.3 \%$ after $4 \mathrm{~h}$ (Fig. $1 ; P=0.005$ by two-way ANOVA).

The extent of improvement in the OTC group (4.5 $\pm 5.8 \%)$ was not significantly related to any of the clinical characteristics listed in Table I. To determine if the observed improvement in flow-mediated dilation with OTC treatment was due to group differences in baseline arterial characteristics, the stimulus for dilation, or an effect of treatment on hemodynamics, we examined baseline vessel diameter, hyperemic response, and hemodynamic parameters both before and after treatment with either OTC or placebo. As shown in Table II, there were no significant differences in these parameters between treatment groups either before or after treatment with OTC or placebo.

To determine if OTC altered smooth muscle cell responsiveness to exogenous NO, we examined brachial artery dilation in response to sublingual nitroglycerin ( $0.4 \mathrm{mg}$; Fig. 2). There was no significant difference in the response to nitroglycerin between the placebo and OTC groups (14.5 \pm 8.2 and

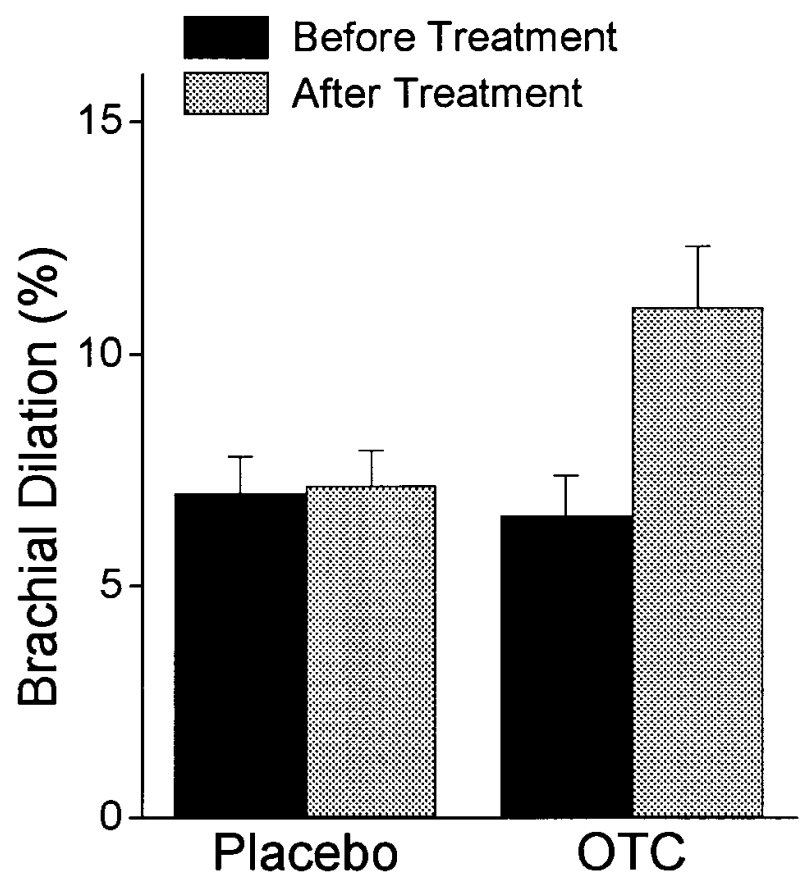

Figure 1. The effect of OTC and placebo on flow-mediated dilation of the brachial artery in patients with CAD. Patients referred for cardiac catheterization underwent brachial ultrasound imaging before (solid bars) and $4 \mathrm{~h}$ after (shaded bars) treatment with either OTC ( 4.5 g orally; $n=24)$ or placebo $(n=24)$ as described in Methods. Data are presented as mean \pm SEM. The effect of OTC was significant by repeated measures ANOVA $(P<0.005)$.

$17.4 \pm 9.3 \%$, respectively; $P=0.32$ ). The study provided sufficient power to detect an increase of $8 \%$ or more in nitroglycerin-induced dilation with $80 \%$ power at the 0.05 significance level. Thus, it is possible that a study with a larger sample size might have demonstrated a significant effect of OTC on the nitroglycerin response.

Plasma antioxidant levels and markers of thiol status. We have previously demonstrated that oral ascorbic acid (2 g) produces acute improvement in flow-mediated dilation of the bra-

Table II. Brachial Artery Flow and Diameter Results

\begin{tabular}{lcc}
\hline \multicolumn{1}{c}{ Parameter } & Placebo & OTC \\
\hline Before treatment & & \\
Diameter (mm) & $4.4 \pm 0.8$ & $4.0 \pm 0.5$ \\
Hyperemic flow (\% increase from baseline) & $600 \pm 340$ & $700 \pm 330$ \\
Heart rate (bpm) & $66 \pm 9$ & $65 \pm 14$ \\
Systolic blood pressure (mmHg) & $135 \pm 20$ & $135 \pm 20$ \\
After treatment & & \\
Diameter (mm) & $4.3 \pm 0.8$ & $4.0 \pm 0.6$ \\
Hyperemic flow (\% increase from baseline) & $590 \pm 450$ & $720 \pm 380$ \\
Pulse (bpm) & $64 \pm 10$ & $69 \pm 14$ \\
Systolic blood pressure (mmHg) & $129 \pm 19$ & $139 \pm 20$ \\
& &
\end{tabular}

Data are presented as mean \pm SD. There were no significant differences in these parameters based upon treatment assignments, and no significant changes after treatment in either treatment group by repeated measures ANOVA. 


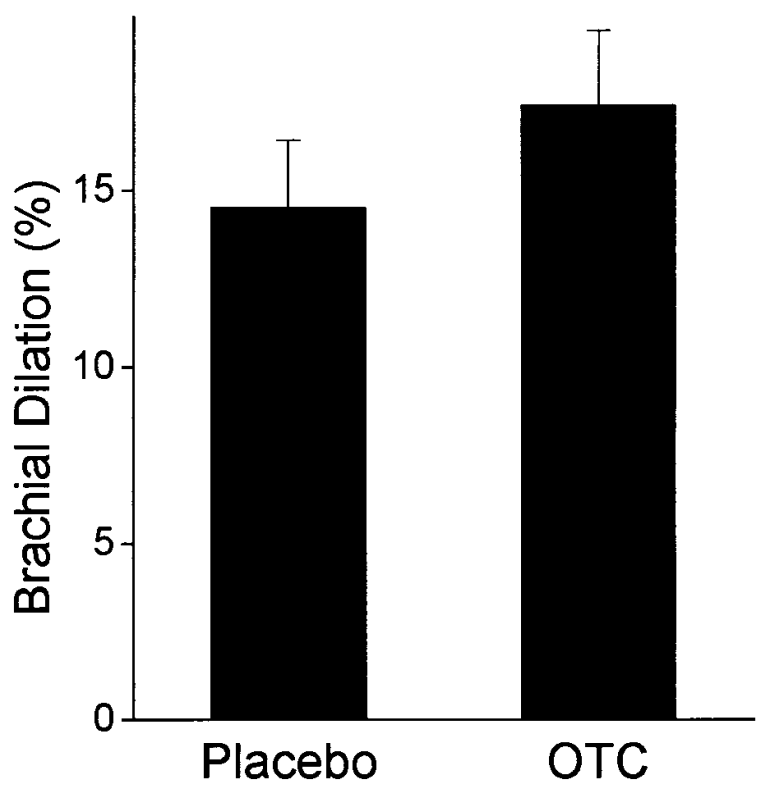

Figure 2. Effect of OTC or placebo on brachial artery dilation to nitroglycerin in patients with CAD. Patients were treated with sublingual nitroglycerin $(0.4 \mathrm{mg}) 10 \mathrm{~min}$ after completion of the experimental protocol described in Fig. 1. Brachial artery images were obtained as described in Methods. Data are presented as mean \pm SEM and are derived from 18 patients treated with placebo and 19 treated with OTC. There was no significant difference between groups $(P=0.32)$.

chial artery in patients with CAD (21). Because oral OTC increases cellular GSH (26) and GSH prevents ascorbic acid oxidation $(36,37)$, we sought to determine if OTC treatment produced any change in plasma ascorbic acid. We did not find any change in plasma ascorbic acid on the basis of treatment with either OTC or placebo (Fig. $3 A ; P=\mathrm{NS}$ ).

To confirm that OTC treatment was effective, we determined whole blood cyst(e)ine concentrations before and after OTC or placebo in a random subset of seven placebo subjects and eight OTC subjects. Treatment with placebo had no effect on whole blood cyst(e)ine $(67 \pm 4$ vs. $68 \pm 4 \mu \mathrm{M})$. In contrast, oral OTC treatment was associated with a near doubling of whole blood cyst(e)ine from $67 \pm 4$ to $124 \pm 9 \mu \mathrm{M}$ (Fig. $3 B ; P<$ 0.001 by repeated measures ANOVA). Since OTC is converted to cysteine by the intracellular enzyme 5-oxoprolinase (23) and not by factors in plasma (27), oral OTC treatment thus resulted in effective intracellular delivery of cysteine.

\section{Discussion}

In this randomized double-blind placebo-controlled study, we found that oral OTC, an agent that increases intracellular GSH in vascular and other tissues $(23,26,27)$, improves endothelium-dependent flow-mediated dilation of the brachial artery in patients with CAD. This improvement in endothelial vasodilator function was not due to an increase in the stimulus for dilation (hyperemia) or any change in the capacity of the artery to dilate to an exogenous source of NO (nitroglycerin). These findings suggest that increasing intracellular GSH levels improves the production and/or bioactivity of EDNO in the brachial artery of patients with documented coronary atherosclerosis.
Considerable controversy exists over the role of intracellular GSH in the action and metabolism of EDNO. Hecker and colleagues found that agonist-induced release of NO from cultured bovine endothelial cells is impaired by alkylating thiols with $N$-ethylmaleimide (NEM) or oxidizing intracellular thiols with 2,2'-dithiodipyridine (DTDP; 38). However, since treatment with DTDP was not associated with a reduction in endothelial cell GSH, those authors concluded that the level of intracellular thiols per se is not important in the release of EDNO. Rather, they implicated the oxidation state of critical thiols involved in $\mathrm{Ca}^{2+}$ homeostasis as the factor responsible for the effects of NEM and DTDP (38). Similar studies with porcine endothelial cells found no correlation between thiol depletion and reduced NO production (39). Mugge and coworkers found that acute $(20-\mathrm{h})$ inhibition of bovine endothelial cell GSH synthesis with L-buthionine- $(S, R)$-sulfoximine had no effect on the release of NO from bovine endothelial cells (40). Taken together, these prior observations suggest that intracellular GSH is not required for bioactive NO production, and would seem at odds with the results presented here.

One possible reconciliation for these seemingly disparate observations may stem from the variety of species involved in these studies. For example, the aforementioned in vitro studies

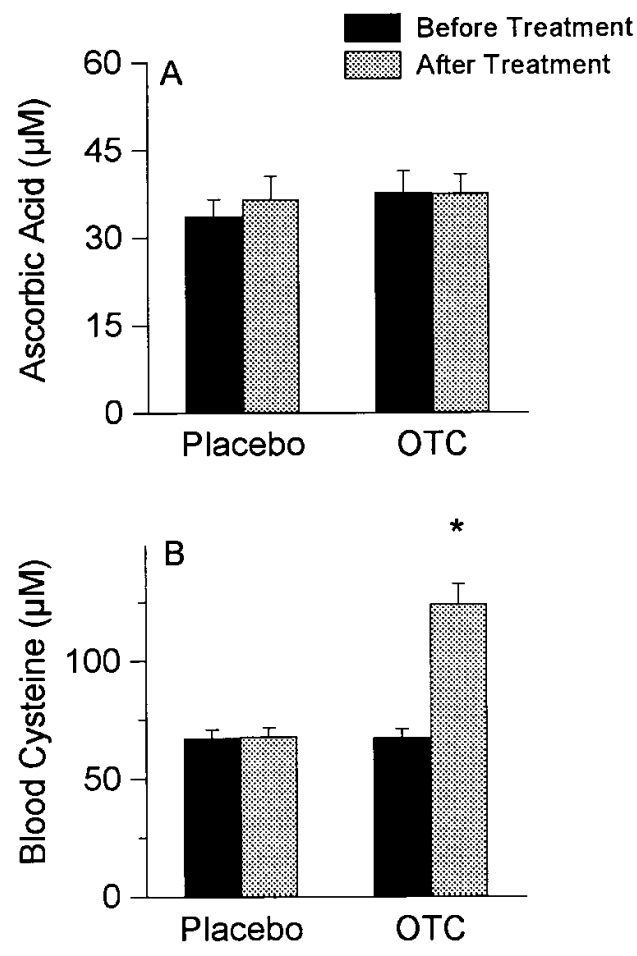

Figure 3. Plasma ascorbic acid and whole blood cyst(e)ine in response to oral OTC or placebo. Plasma and whole blood samples were obtained from patients before and $4 \mathrm{~h}$ after either OTC or placebo. (A) Plasma ascorbic acid was determined in patients receiving either placebo $(n=20)$ or OTC $(n=20)$ as described (34). (B) Whole blood total cyst(e)ine (oxidized + reduced) was determined as described in Methods in randomly selected patients receiving placebo $(n=7)$ or OTC $(n=8)$. Data are mean \pm SEM. The effect of OTC was significant by repeated measures ANOVA $(P<0.001)$. 
used endothelial cells of bovine $(38,40)$ and porcine $(39)$ origin, whereas the results reported here are in humans. In this regard, Ghigo and colleagues (41) used human umbilical vein endothelial cells to study the effect of thiol manipulation on EDNO production. These investigators reduced intracellular GSH levels with 1-chloro-2,4 dinitrobenzene (CDNB), which forms a covalent complex with GSH through glutathione $S$-transferase activity. Treatment of endothelial cells with CDNB was associated with a dose-dependent reduction of both cellular GSH and cellular NO production (41). In addition, bolstering cellular GSH with glutathione monoethyl ester resulted in enhanced NO production, and there was a strong correlation between cellular GSH concentration and ionomycin-induced NO production $(r=0.992)$. These findings are in good agreement with the results reported here, demonstrating that increasing intracellular GSH with OTC improves endothelium-dependent vasodilator function (Fig. 1).

The precise mechanism(s) by which intracellular GSH may modulate the production and/or bioactivity of NO is not yet clear. In isolated enzyme preparations, the neuronal (42) and inducible isoforms (43) of nitric oxide synthase (NOS) both demonstrate optimal enzymatic activity in the presence of GSH. One principal effect of GSH is to prevent inactivation of NOS (43) that is thought to result from the formation of peroxynitrite due to simultaneous generation of NO and superoxide by the enzyme (44). With neuronal NOS, GSH appears to directly stimulate the enzyme $(42,45)$ as well as to inhibit inactivation (45).

Glutathione may also modulate the action and metabolism of EDNO by virtue of its central role in regulation of the intracellular redox state (17). Along with ascorbic acid, GSH is present in millimolar concentrations in the cell cytosol, and serves both to scavenge free radicals and to detoxify reactive oxygen species (17) including peroxynitrite (22). Tetrahydrobiopterin, an essential cofactor for endothelial NOS, is readily oxidized, and both ascorbic acid (46) and thiol compounds (46, 47) have been shown to protect tetrahydrobiopterin from oxidation. Moreover, endothelial L-arginine transport is sensitive to adequate intracellular GSH levels (48). The potential effect(s) of intracellular GSH on cellular tetrahydrobiopterin and L-arginine levels is of particular importance. A relative lack of either tetrahydrobiopterin (49) or L-arginine $(50,51)$ impairs NOS-mediated NO production. There is also evidence that NOS produces superoxide in the absence of L-arginine (50), and superoxide is known to inactivate EDNO (9). Through these indirect effects, GSH has the potential to modulate endothelial cell capacity for NO production.

In addition to $\mathrm{GSH}$, intracellular redox state is also determined in part by ascorbic acid. The status of ascorbic acid and GSH are closely related in vivo. In terms of providing intracellular antioxidant protection, ascorbic acid can replace GSH (52), and GSH can substitute for ascorbic acid (37). This relation between intracellular ascorbic acid and GSH may be relevant for the findings presented here. Using the same patient population as the present study, we previously demonstrated that a single dose of oral ascorbic acid $(2 \mathrm{~g})$ improves brachial artery flow-mediated dilation in $2 \mathrm{~h}$ (21). This effect of ascorbic acid was associated with a twofold increase in plasma ascorbic acid concentration that remained within the normal range (21). In light of these previous findings, one must consider the possibility that increasing intracellular GSH improves EDNO action through sparing intracellular ascorbic acid, al- though direct evidence for such speculation is not available at present.

In addition to its effects on intracellular redox state, GSH may influence the biologic activity of EDNO more directly. For example, in the presence of oxygen (53) or superoxide (54), NO may be oxidized and combine with GSH to form the $S$-nitrosothiol, $S$-nitroso-glutathione (GSNO). GSNO demonstrates biochemical properties that are reminiscent of EDNO, including platelet inhibition and smooth muscle cell relaxation (55). In light of the fact that GSNO demonstrates greater chemical stability than authentic NO (55), it is possible that increasing the intracellular concentration of GSH favors the formation of GSNO, resulting in enhanced bioactivity for EDNO. Such speculation would be consistent with observations that human endothelial cell EDNO action is sensitive to intracellular GSH status (41).

With respect to the formation of $S$-nitrosothiols, we observed that patients treated with OTC demonstrated an increase in whole blood cyst(e)ine, confirming that OTC was absorbed and converted to cysteine in the intracellular space, a process that requires the action of the intracellular enzyme 5-oxoprolinase (23). In vitro studies have shown that reduced thiol enhances the activity of EDNO $(56,57)$. Thus, it is possible that the effect of OTC reported here reflects the enhanced availability of intracellular reduced thiol (cysteine and glutathione) that potentiates the activity of EDNO. Alternatively, one might also argue that the increased availability of extracellular cysteine accounted for our findings. However, we consider this latter possibility unlikely because a recent study demonstrated that an acute infusion $(20 \mathrm{~min})$ of the synthetic thiol $N$-acetylcysteine, which increases plasma cysteine, had no effect on EDNO action in the forearm circulation of normal subjects (58). Previous studies suggest that administration of NAC over this short time course is insufficient to materially increase intracellular glutathione concentration $(24,59)$.

Our study had several limitations. The study involved human subjects, and thus we were unable to directly measure glutathione in arterial tissue and correlate changes in glutathione concentration with changes in EDNO action. However, studies in animals $(25,26)$ clearly show that OTC induces an increase in vascular glutathione concentration, and a prior human study (27) demonstrated that the same dose of OTC increased lymphocyte glutathione concentration over the 4-h time period used in this study. In the present study we examined the effects of intracellular GSH repletion on flow-mediated dilation of the brachial artery, and thus inferences about the potential effects of intracellular GSH on EDNO-mediated control of coronary arterial tone must be made with caution. There are, however, several lines of evidence to suggest that EDNO-mediated control of brachial arterial tone is relevant to the coronary circulation. For example, known risk factors for the clinical expression of coronary artery disease such as hypercholesterolemia, hypertension, diabetes, and tobacco use are associated with abnormal EDNO-mediated vasorelaxation in both brachial (60-62) and coronary arteries (3, 63-65). Moreover, there appears to be a strong correlation between endothelial dysfunction in conduit brachial and coronary arteries of the same patients (66).

In summary, this double-blind placebo-controlled trial demonstrates that oral treatment with OTC, a drug that increases intracellular GSH, is associated with improved EDNO-mediated dilation of the brachial artery in patients with docu- 
mented CAD. This finding suggests that increased intracellular oxidative stress may be an important mechanism for impaired endothelial function in CAD patients. In particular, it appears that intracellular GSH status is an important determinant of endothelial function in patients with angiographically documented CAD.

\section{Acknowledgments}

This work was supported by grants from the American Heart Association, National Center (J.F. Keaney, Jr.), and the National Institutes of Health (HL53398 to J.A. Vita, HL56170 to B. Frei). Joseph A. Vita is an Established Investigator of the American Heart Association, and John F. Keaney, Jr. is the recipient of a Clinical Investigator Development Award (HL03195) from the National Institutes of Health.

\section{References}

1. Vita, J.A., C.B. Treasure, P. Ganz, D.A. Cox, R.D. Fish, and A.P. Selwyn. 1989. Control of shear stress in the epicardial coronary arteries of humans: impairment by atherosclerosis. J. Am. Coll. Cardiol. 14:1193-1199.

2. Ludmer, P.L., A.P. Selwyn, T.L. Shook, R.R. Wayne, G.H. Mudge, R.W. Alexander, and P. Ganz. 1986. Paradoxical vasoconstriction induced by acetylcholine in atherosclerotic coronary arteries. N. Engl. J. Med. 315:1046-1051.

3. Vita, J.A., C.B. Treasure, E.G. Nabel, J.M. McLenachan, R.D. Fish, A.C. Yeung, V.I. Vekshtein, A.P. Selwyn, and P. Ganz. 1990. Coronary vasomotor response to acetylcholine relates to risk factors for coronary artery disease. Circulation. 81:491-497.

4. Levine, G.N., J.F. Keaney, Jr., and J.A. Vita. 1995. Cholesterol reduction in cardiovascular disease: Clinical benefits and possible mechanisms. N. Engl. J. Med. 332:512-521.

5. Keaney, J.F., and J.A. Vita. 1995. Atherosclerosis, oxidative stress and antioxidant protection in endothelium-derived relaxing factor action. Prog. Cardiovasc. Dis. 38:129-154.

6. Ohara, Y., T.E. Peterson, and D.G. Harrison. 1993. Hypercholesterolemia increases endothelial superoxide anion production. J. Clin. Invest. 91: 2546-2551.

7. Keaney, J.F., Jr., A. Xu, D.C. Cunningham, T. Jackson, B. Frei, and J.A. Vita. 1995. Dietary probucol preserves endothelial function in cholesterol-fed rabbits by limiting vascular oxidative stress and superoxide production. J. Clin. Invest. 95:2520-2529.

8. Rubanyi, G.M., and P.M. Vanhoutte. 1986. Superoxide anions and hyperoxia inactivate endothelium-derived relaxing factor. Am. J. Physiol. 250:H822H827.

9. Gryglewski, R.J., R.M. Palmer, and S. Moncada. 1986. Superoxide anion is involved in the breakdown of endothelium-derived vascular relaxing factor. Nature. 320:454-456.

10. Keaney, J.F., Jr., and B. Frei. 1994. Antioxidant protection of low-density lipoprotein and its role in the prevention of atherosclerotic vascular disease. In Natural Antioxidants in Human Health and Disease. B. Frei, editor. Academic Press, Inc. San Diego. 303-352.

11. Kugiyama, K., S.A. Kerns, J.D. Morrisett, R. Roberts, and P.D. Henry. 1990. Impairment of endothelium-dependent arterial relaxation by lysolecithin in modified low-density lipoproteins. Nature. 344:160-162.

12. Chin, J.H., S. Azhar, and B.B. Hoffman. 1992. Inactivation of endothelium-derived relaxing factor by oxidized lipoproteins. J. Clin. Invest. 89:10-18.

13. Mugge, A., J.H. Elwell, T.E. Peterson, T.G. Hofmeyer, D.D. Heistad, and D.G. Harrison. 1991. Chronic treatment with polyethylene-glycolated superoxide dismutase partially restores endothelium-dependent vascular relaxations in cholesterol-fed rabbits. Circ. Res. 69:1293-1300.

14. Keaney, J.F., Jr., J.M. Gaziano, A. Xu, B. Frei, J. Curran-Celantano, G.T. Shwaery, J. Loscalzo, and J.A. Vita. 1993. Dietary antioxidants preserve endothelium-dependent vessel relaxation in cholesterol-fed rabbits. Proc. Natl. Acad. Sci. USA. 90:11880-11884.

15. Simon, B.C., C.C. Haudenschild, and R.A. Cohen. 1993. Preservation of endothelium-dependent relaxation in atherosclerotic rabbit aorta by probucol. J. Cardiovasc. Pharmacol. 21:893-901.

16. Anderson, T.J., I.T. Meredith, A.C. Yeung, B. Frei, A. Selwyn, and P. Ganz. 1995. The effect of cholesterol lowering and antioxidant therapy on endothelium-dependent coronary vasomotion. N. Engl. J. Med. 332:488-493.

17. Meister, A. 1994. Glutathione-ascorbic acid antioxidant system in animals. J. Biol. Chem. 269:9397-9400.

18. Ting, H.H., F.K. Timimi, K.S. Boles, S.J. Creager, P. Ganz, and M.A. Creager. 1996. Vitamin C improves endothelium-dependent vasodilation in patients with non-insulin-dependent diabetes mellitus. J. Clin. Invest. 97:22-28.
19. Heitzer, T., H. Just, and T. Munzel. 1996. Antioxidant vitamin C improves endothelial dysfunction on chronic smokers. Circulation. 94:6-9.

20. Ting, H.H., F.K. Timimi, E.A. Haley, M.A. Roddy, P. Ganz, and M.A Creager. 1997. Vitamin C improves endothelium-dependent vasodilation in forearm resistance vessels of humans with hypercholesterolemia. Circulation. 95:2617-2622.

21. Levine, G.N., B. Frei, S.N. Koulouris, M.D. Gerhard, J.F. Keaney, Jr., and J.A. Vita. 1996. Ascorbic acid reverses endothelial vasomotor dysfunction in patients with coronary artery disease. Circulation. 96:1107-1113.

22. Ma, X.L., B.L. Lopez, G.L. Liu, T.A. Christopher, F. Gao, Y. Guo, G.Z Feuerstein, R.R. Ruffolo Jr., F.C. Barone, and T.L. Yue. 1997. Hypercholesterolemia impairs a detoxification mechanism against peroxynitrite and renders the vascular tissue more susceptible to oxidative injury. Circ. Res. 80:894-901.

23. Williamson, J.M., and A. Meister. 1981. Stimulation of hepatic glutathione formation by administration of L-2-oxothiazolidine-4-carboxylate: a 5-oxo-L-prolinase substrate. Proc. Natl. Acad. Sci. USA. 78:936-939.

24. Williamson, J.M., B. Boettcher, and A. Meister. 1982. Intracellular cysteine delivery system that protects against toxicity by promoting glutathione synthesis. Proc. Natl. Acad. Sci. USA. 79:6246-6249.

25. Boesgaard, S., H.E. Poulsen, J. Aldershvile, S. Loft, M.E. Anderson, and A. Meister. 1993. Acute effects of nitroglycerin depend on both plasma and intracellular sulfhydryl compound levels in vivo: effects of agents with different sulfhydryl modulating properties. Circulation. 87:547-553.

26. Boesgaard, S., J. Aldershvile, H.E. Poulsen, S. Loft, M.E. Anderson, and A. Meister. 1994. Nitrate tolerance in vivo is not associated with depletion of arterial or venous thiol levels. Circ. Res. 74:115-120.

27. Porta, P., S. Aebi, K. Summer, and B.H. Lauterburg. 1991. L-2-oxothiazolidine-4-carboxylic acid, a cysteine prodrug: Pharmacokinetics and effects on thiols in plasma and lymphocytes in humans. J. Pharmacol. Exp. Ther. 257:331-334

28. Hamsten, A., G. Walldius, A. Szamosi, G. Dahlen, and U. deFaire. 1986. Relationship of angiographically defined coronary artery disease to serum lipoproteins and apolipoproteins in young survivors of myocardial infarction. Circulation. 73:1097-1110.

29. Lieberman, E.H., M.D. Gerhard, A. Uehata, B.W. Walsh, A.P. Selwyn, P. Ganz, A.C. Yeung, and M.A. Creager. 1994. Estrogen improves endothelium-dependent, flow mediated vasodilation in post menopausal women. Ann. Intern. Med 121:936-941.

30. Lieberman, E.H., M.D. Gerhard, A. Uehata, A.P. Selwyn, P. Ganz, A.C. Yeung, and M.A. Creager. 1996. Flow-induced vasodilation of the human brachial artery is impaired in patients $<40$ years of age with coronary artery disease. Am. J. Cardiol. 78:1210-1214.

31. Vita, J.A., and J.F. Keaney, Jr. 1996. Ultrasound assessment of endothelial vasomotor function. In Diagnostics of Vascular Diseases: Principals and Technology. P. Lanzer, and M. Lipton, editors. Springer-Verlag, Berlin. 249-259.

32. Assman, G., H. Schreiwer, G. Schmitz, and E.O. Hagele. 1983. Quantification of high-density-lipoprotein cholesterol by precipitation with phosphotungstic acid/MgCl2. Clin. Chem. 29:2026-2030.

33. Friedewald, W.T., R.I. Levy, and D.S. Fredrickson. 1972. Estimation of the concentration of low-density lipoprotein cholesterol in plasma, without use of the preparative ultracentrifuge. Clin. Chem. 18:499-502.

34. Frei, B., L. England, and B.N. Ames. 1989. Ascorbate is an outstanding antioxidant in human blood plasma. Proc. Natl. Acad. Sci. USA. 86:6377-6381.

35. Fiskerstrand, T., H. Refsum, G. Kvalheim, and P.M. Ueland. 1993. Homocysteine and other thiols in plasma and urine: automated determination and sample stability. Clin. Chem. 39:263-271.

36. Winkler, B.S. 1987. In vitro oxidation of ascorbic acid and its prevention by GSH. Biochim. Biophys. Acta. 925:258-264.

37. Martensson, J.J., E.W. Han, A. Griffith, and A. Meister. 1993. Glutathione delays the onset of scurvy in ascorbate-deficient guinea pigs. Proc Natl. Acad. Sci. USA. 90:317-321.

38. Hecker, M., I. Siegle, H. Macarthur, W.C. Sessa, and J.R. Vane. 1992 Role of intracellular thiols in release of EDRF from cultured endothelial cells Am. J. Physiol. 262:H888-H896.

39. Murphy, M.E., H.-M. Piper, H. Watanabe, and H. Sies. 1991. Nitric oxide production by cultured aortic endothelial cells in response to thiol depletion and replenishment. J. Biol. Chem. 266:19378-19383.

40. Mugge, A., J.K. Elwell, T.E. Peterson, and D.G. Harrison. 1991. Release of intact endothelium-derived relaxing factor depends on endothelial superoxide dismutase activity. Am. J. Physiol. 260:C219-C225.

41. Ghigo, D., P. Alessio, A. Foco, F. Bussolino, C. Costamagna, R. Heller, G. Garbarino, G.P. Pescarmona, and A. Bosia. 1993. Nitric oxide synthesis is impaired in glutathione-depleted human umbilical vein endothelial cells. Am. J. Physiol. 265:C728-C732.

42. Komori, Y., J. Hyun, K. Chiang, and J.M. Fukuto. 1995. The role of thiols in the apparent activation of rat brain nitric oxide synthase (NOS). J. Biochem. 117:923-927.

43. Stuehr, D.J., N.S. Kwon, and C.F. Nathan. 1990. FAD and GSH participate in macrophage synthesis of nitric oxide. Biochem. Biophys. Res. Commun 168:558-565.

44. Hobbs, A.J., J.M. Fukoto, and L.J. Ignarro. 1994. Formation of free nitric oxide from L-arginine by nitric oxide synthase: direct enhancement of generation by superoxide dismutase. Proc. Natl. Acad. Sci. USA. 91:10992-10996. 
45. Hofman, H., and H.H.W. Schmidt. 1995. Thiol dependence of nitric oxide synthase. Biochemistry. 34:13443-13452.

46. Howells, D.W., and K. Hyland. 1987. Direct analysis of tetrahydrobiopterin in cerebrospinal fluid by high-performance liquid chromatography with redox electrochemistry: prevention of autooxidation during storage and analysis. Clin. Chim. Acta. 167:23-30.

47. Heales, S., and K. Hyland. 1989. Determination of quinonoid dihydrobiopterin by high-performance liquid chromatography and electrochemical detection. J. Chromatogr. 494:77-85.

48. Patel, J.M., A.J. Abeles, and E.R. Block. 1996. Nitric oxide exposure and sulfhydryl modulation alter L-arginine transport in cultured pulmonary artery endothelial cells. Free Radical Biol. Med. 20:629-637.

49. Heinzel, B., M. John, P. Klatt, E. Böhme, and B. Mayer. 1992. Ca2+/ calmodulin-dependent formation of hydrogen peroxide by brain nitric oxide synthase. Biochem. J. 281:627-630.

50. Pou, S., W.S. Pou, D.S. Bredt, S.H. Snyder, and G.M. Rosen. 1992. Generation of superoxide by purified brain nitric oxide synthase. J. Biol. Chem. 267: 24173-24176.

51. Xia, Y., V.L. Dawson, T.M. Dawson, S.H. Snyder, and J.L. Zweier. 1996. Nitric oxide synthase generates superoxide and nitric oxide in argininedepleted cells leading to peroxynitrite-mediated cell injury. Proc. Natl. Acad. Sci. USA. 93:6770-6774.

52. Martensson, J., and A. Meister. 1991. Glutathione deficiency decreases tissue ascorbate levels in newborn rats: Ascorbate spares glutathione and protects. Proc. Natl. Acad. Sci. USA. 88:4656-4660.

53. Wink, D.A., R.W. Nims, J.F. Darbyshire, D. Christodoulou, I. Hanbauer, G.W. Cox, F. Laval, J. Laval, J.A. Cook, and M.C. Krishna. 1994. Reaction kinetics for nitrosation of cysteine and glutathione in aerobic nitric oxide solutions at neutral $\mathrm{pH}$. Insights into the fate and physiological effects of intermediates generated in the NO/O2 reaction. Chemical Res. Toxicol. 7:519-525.

54. Moro, M.A., V. Darley-Usmar, D.A. Goodwin, N.G. Read, R. ZamoraPino, M. Feelisch, M.W. Radomski, and S. Moncada. 1994. Paradoxical fate and biological action of peroxynitrite on human platelets. Proc. Natl. Acad. Sci. USA. 91:6702-6706.

55. Stamler, J.S., D.J. Singel, and J. Loscalzo. 1992. Biochemistry of nitric oxide and its redox-activated forms. Science. 258:1898-1902.

56. Stamler, J.S., M.E. Mendelsohn, P. Amarante, D. Smick, N. Andon, P.F. Davies, J.P. Cooke, and J. Loscalzo. 1989. N-acetylcysteine potentiates platelet inhibition by endothelium-derived relaxing factor. Circ. Res. 65:789-795.

57. Cooke, J.P., J. Stamler, N. Andon, P.F. Davies, G. McKinley, and J. Loscalzo. 1990. Flow stimulates endothelial cells to release nitrovasodilator that is potentiated by reduced thiol. Am. J. Physiol. 259(Pt. 2):H804-H812.

58. Creager, M.A., M.A. Roddy, K. Boles, and J.S. Stamler. 1997. N-acetylcysteine does not influence the activity of endothelium-derived relaxing factor in vivo. Hypertension. 29:668-672.

59. Wong, B.K., H.C. Chan, and G.B. Corcoran. 1986. Selective effects of $\mathrm{N}$-acetylcysteine stereoisomers on hepatic glutathione and plasma sulfate in mice. Toxicol. Appl. Pharmacol. 86:421-429.

60. Celermajer, D.S., K.E. Sorensen, V.M. Gooch, D.J. Spiegelhalter, O.I Miller, I.D. Sullivan, J.K. Lloyd, and J.E. Deanfield. 1992. Non-invasive detection of endothelial dysfunction in children and adults at risk of atherosclerosis. Lancet. 340:1111-1115.

61. Sorenson, K.E., D.S. Celermajer, and D. Georgakopoulos. 1994. Impairment of endothelium-dependent dilation is an early event in children with familial hypercholesterolemia and is related to the lipoprotein (a) level. J. Clin. Invest. 93:50-55.

62. Celermajer, D.S., K.E. Sorensen, C. Bull, J. Robinson, and J.E. Deanfield. 1994. Endothelium-dependent dilation in the systemic arteries of asymptomatic subject relates to coronary risk factors and their interaction. J. Am. Coll. Cardiol. 24:1468-1474.

63. Treasure, C.B., S.V. Manoukian, J.L. Klein, J.A. Vita, E.G. Nabel, G.H Renwick, A.P. Selwyn, R.W. Alexander, and P. Ganz. 1992. Epicardial coronary artery responses to acetylcholine are impaired in hypertensive patients. Circ. Res. 71:776-781

64. Nitenberg, A., P. Valersi, R. Sachs, M. Dali, E. Aptecar, and J.R. Attali. 1993. Impairment of coronary vascular reserve in and ACH-induced coronary vasodilation in diabetic patients with angiographically normal coronary arteries and normal left ventricular systolic function. Diabetes. 42:1017-1025.

65. Nitenberg, A., I. Antony, and J.M. Foult. 1993. Acetylcholine-induced coronary vasoconstriction in young, heavy smokers with normal coronary arteriographic findings. Am. J. Med. 95:71-77.

66. Anderson, T.J., A. Uehata, M.D. Gerhard, I.T. Meredith, S. Knab, D. Delagrange, E. Leiberman, P. Ganz, M.A. Creager, A.C. Yeung, and A.P. Selwyn. 1995. Close relation of endothelial function in the human coronary and peripheral circulations. J. Am. Coll. Cardiol. 26:1235-1241. 